4. When the superficial inguinal lymph nodes are retracted over the facia lata from the craniolateral part, the fossa ovalis is identified at the central part of the incision, which is covered by the cribriform fascia. Besides, during the excision of the superficial nodes at the caudal part of this area, the great saphenous vein passing through the fossa ovalis is identified.

5. After total excision of the superficial inguinal lymph nodes, the cribriform fascia is dissected. The femoral vein is identified below the fascia lata, covered by the femoral sheath between the sartorius muscle laterally and the adductor longus muscle medially. The great saphenous vein drains into the femoral vein, and the deep inguinofemoral lymph nodes, which are located medial to the femoral vein, are dissected. The femoral artery lies at the lateral part of the femoral vein, and the femoral nerve is the most lateral component in the femoral sheath.

6. The deep inguinofemoral lymph nodes cover the base of the great saphenous vein.

7. During excision of the deep inguinofemoral lymph nodes, the deep external pudendal vein should also be kept in mind, which drains into the great saphenous or femoral vein. Conclusion* The essential anatomy of the superficial inguinal and deep femoral lymph nodes, with Scarpa's fascia, facia lata of the thigh, and cribriform fascia should be known to perform an inguinofemoral lymphadenectomy.

\section{Late breaking abstracts}

\section{Cervical cancer}

\section{KEYNOTE-826: PEMBROLIZUMAB PLUS CHEMOTHERAPY VERSUS PLACEBO PLUS CHEMOTHERAPY FOR PERSISTENT, RECURRENT, OR METASTATIC CERVICAL CANCER}

${ }^{1} \mathrm{~N}$ Colombo*, ${ }^{2} \mathrm{C}$ Dubot, ${ }^{3} \mathrm{D}$ Lorusso, ${ }^{4} \mathrm{~V}$ Caceres, ${ }^{5} \mathrm{~K}$ Hasegawa, ${ }^{6} \mathrm{R}$ Shapira-Frommer ${ }^{7} \mathrm{~K}$ Tewari, ${ }^{8} \mathrm{P}$ Salman, ${ }^{9} \mathrm{E}$ Hoyos Usta, ${ }^{10} \mathrm{E}$ Yañez, ${ }^{11} \mathrm{M}$ Gümüş, ${ }^{12} \mathrm{M}$ Olivera Hurtado de Mendoza, ${ }^{13} \mathrm{~V}$ Samouëlian, ${ }^{14} \mathrm{~V}$ Castonguay, ${ }^{15} \mathrm{~A}$ Arkhipov, ${ }^{16} \mathrm{~S}$ Toker, ${ }^{16} \mathrm{~K} \mathrm{LI}$, ${ }^{16} \mathrm{~S}$ Keefe, ${ }^{17} \mathrm{~B}$ Monk. 'University of Milan-Bicocca and European Institute of Oncology (IEO) IRCCS, Milan, Italy; ${ }^{2}$ Institut Curie Saint-Cloud, Group d'Investigateurs Nationaux pour l'Etude des Cancers Ovariens (GINECO), Saint-Cloud, France; ${ }^{3}$ Fondazione Policlinico Universitario A Gemelli IRCCS and Catholic University of Sacred Heart, Rome, Italy; ${ }^{4}$ Institute of Oncology Angel H. Roffo, Buenos Aires, Argentina; ${ }^{5}$ Saitama Medical University International Medical Center, Hidaka, Japan; ${ }^{6}$ Ella Lemelbaum Institute for Immuno-Oncology, Sheba Medical Center, Ramat Gan, Israel; ${ }^{7}$ University of California Irvine, Irvine, USA; ${ }^{8}$ Oncovida Cancer Center, Providencia, Chile; ${ }^{9}$ mat Oncomedica S.A., Montería, Colombia; ${ }^{10}$ Universidad de La Frontera, Temuco, Chile; "IIstanbul Medeniyet University Hospital, Istanbul, Turkey; ${ }^{12}$ Instituto Nacional de Enfermedades Neoplásicas, Medical Oncology, Lima, Peru; ${ }^{13}$ Centre Hospitalier de I'Université de Montréal (CHUM), Centre de Recherche de I'Université de Montréal (CRCHUM), Université de Montréal, Montreal, Canada; ${ }^{14}$ Centre Hospitalier Universitaire de Québec, Université Laval, Québec City, Canada; ${ }^{15}$ Medical Rehabilitation Center under the Ministry of Health of Russian Federation, Moscow, Russian Federation; ${ }^{16}$ Merck and Co., Inc., Kenilworth, USA; ${ }^{17}$ Arizona Oncology (US Oncology Network), University of Arizona College of Medicine, Creighton University School of Medicine, Phoenix, USA

\subsection{6/ijgc-2021-ESG0.641}

Introduction/Background* Pembrolizumab has efficacy in previously treated, PD-L1-positive advanced cervical cancer. KEYNOTE-826 (NCT03635567) was a phase 3, randomised, double-blind trial of pembrolizumab or placebo added to chemotherapy \pm bevacizumab for first-line treatment of recurrent, persistent, or metastatic cervical cancer.
Methodology Eligible adults had persistent, recurrent, or metastatic cervical cancer not previously treated with systemic chemotherapy and not amenable to curative treatment. Patients were randomised 1:1 to pembrolizumab $200 \mathrm{mg}$ or placebo Q3W for $\leq 35$ cycles added to chemotherapy (paclitaxel plus cisplatin or carboplatin) \pm bevacizumab and stratified by metastatic status at diagnosis, planned bevacizumab use, and PD-L1 combined positive score (CPS). Dual primary endpoints were PFS (RECIST v1.1, investigator review) and OS tested sequentially in the CPS $\geq 1$, all-comer, and CPS $\geq 10$ populations.

Result(s)* 617 patients were randomized: 308 to pembrolizumab plus chemotherapy (63.6\% with bevacizumab) and 309 to placebo plus chemotherapy (62.5\% with bevacizumab); 548 (88.8\%) patients had CPS $\geq 1,317$ (51.4\%) had CPS $\geq 10$. At the protocol-specified first interim analysis, pembrolizumab plus chemotherapy \pm bevacizumab significantly improved PFS in the CPS $\geq 1$ (median, 10.4 vs 8.2 months; HR, 0.62 [95\% CI, 0.50-0.77]; $\mathrm{P}<0.001)$, all-comer $(10.4$ vs 8.2 months; $0.65[0.53-0.79] ; \mathrm{P}<0.001)$, and CPS $\geq 10 \quad(10.4$ vs 8.1 months; 0.58 [0.44-0.77]; $\mathrm{P}<0.001)$ populations. OS was also significantly improved in the CPS $\geq 1$ (median, not reached $[\mathrm{NR}]$ vs 16.3 months; HR, 0.64 [95\% CI, 0.50-0.81]; $\mathrm{P}<0.001)$, all-comer (24.4 vs 16.5 months; 0.67 [0.54-0.84]; $\mathrm{P}<0.001$ ), and CPS $\geq 10$ (NR vs 16.4 months; 0.61 [0.440.84]; $\mathrm{P}=0.001$ ) populations. Benefits were seen in the with and without bevacizumab subgroups. The incidence of grade $\geq 3$ AEs was $81.8 \%$ in the pembrolizumab arm and $75.1 \%$ in the placebo arm. Anaemia and neutropenia were the most common grade $\geq 3$ AEs $(30.3 \%$ vs $26.9 \%$ and $12.4 \%$ vs 9.7\%, respectively).

Conclusion* Pembrolizumab plus chemotherapy \pm bevacizumab significantly improves OS and PFS in patients with persistent, recurrent, or metastatic cervical cancer. Along with a manageable safety profile, the clinically meaningful survival benefits suggest pembrolizumab plus chemotherapy \pm bevacizumab may be a new standard first-line therapy for this population.

\section{IDENTIFYING PREDICTORS OF LYMPH NODE METASTASES IN EARLY-STAGE CERVICAL CANCER BY TRANSFERRING PREDICTION MODELS ACROSS INTERNATIONAL REGISTRIES}

${ }^{1} T$ Schnack, ${ }^{2 ; 3} \mathrm{H}$ Wenzel ${ }^{*},{ }^{4 ; 5} \mathrm{~A}$ Norberg Hardie, ${ }^{6 ; 7} \mathrm{R}$ Bekkers, ${ }^{4 ; 5} \mathrm{H}$ Falconer, ${ }^{1} \mathrm{C}$ Høgdall, ${ }^{8 ; 9 ; 10} \mathrm{P}$ Jensen, ${ }^{2 ; 11} \mathrm{~V}$ Lemmens, ${ }^{2} \mathrm{~F}$ Martin, ${ }^{3} \mathrm{H}$ Nijman, ${ }^{2} \mathrm{M}$ Van der $\mathrm{Aa},{ }^{2} \mathrm{~A}$ Moncada-Torres. 'Juliane Marie Centre, Rigshospitalet, University of Copenhagen, Department of Gynaecology, Copenhagen, Denmark; ${ }^{2}$ Netherlands Comprehensive Cancer Organisation (IKNL), Department of Research and Development, Utrecht, Netherlands; ${ }^{3}$ University Medical Centre Groningen, University of Groningen, Department of Obstetrics and Gynaecology, Groningen, Netherlands; ${ }^{4}$ Karolinska University Hospital, Department of Pelvic Cancer, Stockholm, Sweden; ${ }^{5}$ Karolinska Institutet, Department of Women's and Children's Health, Stockholm, Sweden; ${ }^{6}$ GROW school for oncology and developmental biology, Maastricht University Medical Centre+, Department of Obstetrics and Gynaecology, Maastricht, Netherlands; ${ }^{7}$ Catharina Hospital, Department of Obstetrics and Gynaecology, Eindhoven, Netherlands; ${ }^{8}$ Aarhus University Hospital, Department of Gynaecology, Aarhus, Denmark; ${ }^{9}$ Aarhus University, Institute of Clinical Medicine, Faculty of Health, Aarhus, Denmark; ${ }^{10}$ University of Southern Denmark, Clinical Institute, Faculty of Health Sciences, Odense, Denmark; ${ }^{11}$ Erasmus MC University Medical Centre, Department of Public Health, Rotterdam, Netherlands

\subsection{6/ijgc-2021-ESG0.642}

Introduction/Background* Identifying risk factors for lymph node metastases in early-stage cervical cancer may aid in tailoring treatment. Unfortunately, early-stage cervical cancer research is often hampered by small sample sizes. 
A natural solution to increase sample size is to incorporate data from multiple sources, traditionally done through centralization. Unfortunately, this approach presents disadvantages concerning privacy protection. Alternatively, models can be trained in one source and tested in another setting, sharing only the model coefficients (figure 1). This approach does not disclose any privacy-sensitive information. Additionally, external validation provides stronger evidence on the generalizability of the obtained statistical model.

In this study, we used the alternative approach to increase sample size and identify risk factors for postoperative lymph node metastases ( $\mathrm{pN} 1)$ in early-stage cervical cancer.

Methodology We conducted an international observational cohort study using data from the Danish Gynaecologic Cancer Database (DGCD) and the Netherlands Cancer Registry (NCR). We included women diagnosed with early-stage cervical squamous cell carcinoma, adenocarcinoma or adenosquamous carcinoma between 2005-2017, who were treated with radical surgery.

We generated two logistic regression models to identify risk factors for pN1. Model A was trained on NCR data and tested with DGCD data, while model B was obtained the

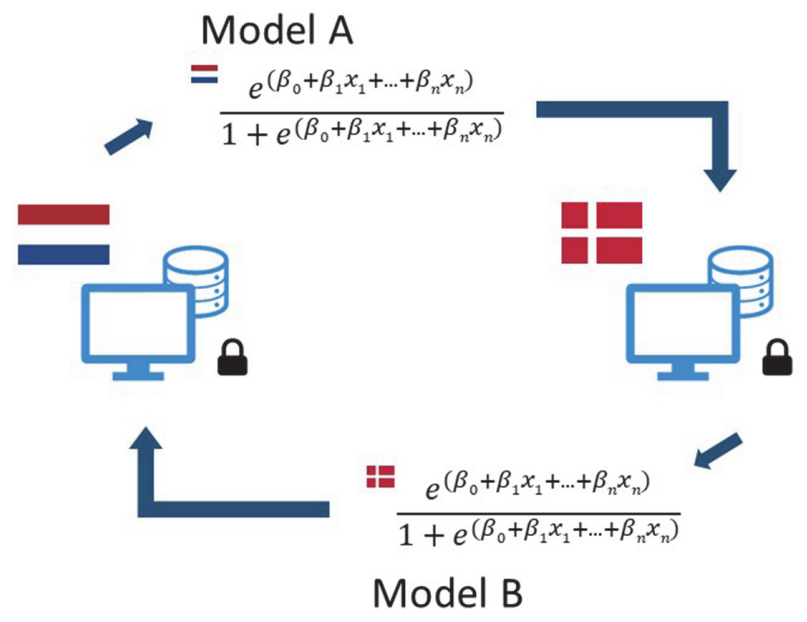

Abstract 1180 Figure 1 A privacy-preserving alternative to small sample analysis. Model A is trained on data from the NCR and tested on DGCD data. Model B was obtained the other way around. Only model coefficients need to be shared and, thereby, no privacy-sensitive information is disclosed
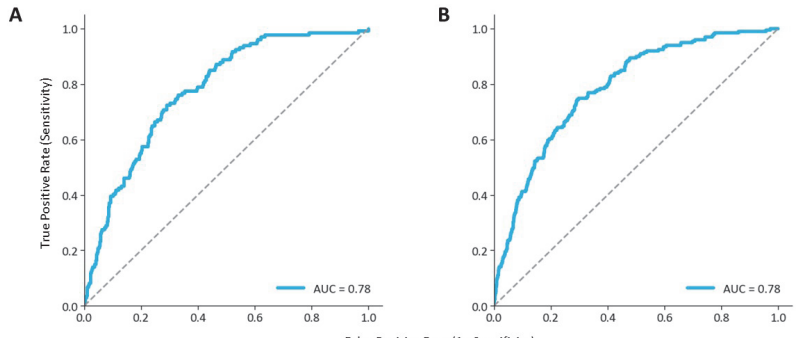

Abstract 1180 Figure 2 Areas under the curve for two models identififying risk factors for postoperative lymph node metastases in earl-stage cervical cancer. 2A: Model A trained with NCR data, tested on DGCD data; 2B: Model B trained with DGCD data, tested on NCR data. other way around. The performance of each model was assessed using areas under the curve (AUCs).

Result(s)* A total of 2735 patients were included in the analyses.

In model A - trained on data from 1476 Dutch patients and tested with data from 1259 Danish patients - tumour size (odds ratio 1.62; 95\% confidence interval [1.37-1.91]), depth of invasion (1.30 [1.12-1.53]) and LVSI (5.13 [3.50-7.52]) were identified as risk factors for $\mathrm{pN} 1$, and body mass index as protective factor $(0.80$ [0.67-0.95].

In model B, trained on Danish data $(n=1259)$ and tested with Dutch data $(n=1476)$, tumour size (1.48 [1.23-1.79]), depth of invasion (1.27 [1.04-1.54]) and LVSI (3.85 [2.535.86]) were associated with increased risk of $\mathrm{pN} 1$.

External validation of model $\mathrm{A}$ and $\mathrm{B}$ both showed AUCs of 0.78 (figure 2).

Conclusion* Tumour size, depth of invasion and LVSI were identified as risk factors for $\mathrm{pN} 1$ in both models.

Separate models, containing either Danish or Dutch data have shown to be valid by privacy-preserving external validation.

\section{RESECTION OF THE UPPER PARACERVICAL LYMPHOVASCULAR TISSUE SHOULD BE A PART OF A PELVIC SENTINEL LYMPH NODE ALGORITHM IN EARLY STAGE CERVICAL CANCER}

O Lührs*, L Ekdahl, B Geppert, C Lönnerfors, J Persson. Lund University Faculty of Medicine, Department of Obstetrics and Gynecology, Lund, Sweden

\subsection{6/ijgc-2021-ESG0.643}

Introduction/Background* To investigate the prevalence of lymph nodes and lymph node metastases (LNMs) in the upper paracervical lymphovascular tissue (UPLT) in early stage cervical cancer.

Methodology In this prospective study consecutive women with stage IA1-IB1 cervical cancer underwent a pelvic lymphadenectomy including identification of sentinel nodes (SLNs) as part of a nodal staging procedure in conjunction with a robotic radical hysterectomy $(\mathrm{RRH})$ or robotic radical trachelectomy (RRT). Indocyanine green (ICG) was used as tracer. The UPLT was separately removed and defined as "SLN-parametrium" and, as all SLN tissue, subjected to ultrastaging and immunohistochemistry. Primary endpoint was prevalence of lymph nodes and metastatic lymph nodes in the UPLT. Secondary endpoints were complications associated with removal of the UPLT.

Result(s)* One hundred and forty-five women were analysed. Nineteen (13.1\%) had pelvic LNMs, all identified by at least one metastatic SLN. In 76 women (52.4\%) at least one UPLT lymph node was identified. Metastatic UPLT lymph nodes were identified in six women of which in three women $(2.1 \%$ of all women and $15.8 \%$ of node positive women) without lateral pelvic LNMs. Thirteen women had lateral pelvic SLN LNMs with either no $(n=5)$ or benign $(n=8)$ UPLT lymph nodes. No intraoperative complications occurred due to the removal of the UPLT.

Conclusion* Removal of the UPLT should be an integral part of the SLN concept in early stage cervical cancer. 